\title{
Psychoanalytische Psychotherapie und die «European Federation for Psychoanalytic Psychotherapy in the Public Sector» (EFPP)
}

\author{
R. Balmer, K. Kraan, J. Pestalozzi, M. Schneider
}

Die Psychotherapie nimmt innerhalb der medizinischen Versorgung zunehmend eine wichtige Stelle ein. Sie muss sich dabei auf die komplexen psychosozialen Bedingungen verschiedenster Patienten ausrichten. Dieser Herausforderung hat sich die EFPP gestellt, deshalb wird sie hier kurz portraitiert.

\section{Einleitung}

Im folgenden soll die Psychoanalytische Psychotherapie kurz beschrieben und die europäische Organisation, die sie vertritt, vorgestellt werden. Die Psychoanalyse wurde seit ihrer Entdeckung immer wieder heftig kritisiert, und in den letzten Jahren ist sie als Psychotherapieform durch andere Methoden auch stark konkurrenziert worden. Sie musste sich mit umfassenden gesellschaftlichen Veränderungen (z.B. andere Familienstrukturen, Migration usw.) und mit den damit verbundenen neuen oder vermehrt auftretenden Krankheitsbildern (z.B. narzisstische und Borderline-Persönlichkeitsstörungen) klinisch und theoretisch, aber v.a. auch praktischtherapeutisch auseinandersetzen.

Die Psychoanalytische Psychotherapie ist demnach angewandte, d.h. an die soziale und klinische Situation angepasste Psychoanalyse. Sie wird meist im Sitzen durchgeführt, und die Stundenfrequenz pro Woche liegt bei 1-3 Stunden, d.h. im Durchschnitt bei 2 Wochenstunden. Häufig sind auch andere Modifikationen des Rahmens nötig und auch die Pharmakotherapie ist oft Bestandteil dieser Behandlungsform.

Die wichtigsten Ziele der Behandlung bleiben aber die längerfristige Veränderung der Persönlichkeitsstruktur und die Behebung der damit verbundenen Beziehungsstörung, die sich in psychiatrischen Symptomen manifestiert. Struktur- und Beziehungsstörungen werden in der psychoanalytischen Psychotherapie in der Übertragung, d.h. in der Arzt-Patient-Beziehung, behandelt. Die lange und aufwendige Ausbildung analytischer Psychotherapeuten ist gerade deshalb notwendig, weil es viel Erfahrung braucht, um diese weitgehend unbewussten Prozesse $\mathrm{zu}$ verstehen und um zum Patienten eine hilfreiche therapeutische Beziehung herzustellen, die nachhaltige Veränderung erst möglich macht. Längerfristige psychoanalytische Psychotherapien unterscheiden sich, wie neuere Studien zeigen, durch ihre Nachhaltigkeit von vielen anderen, kurzfristigeren psychotherapeutischen Methoden.

\section{Die europäische Vereinigung: EFPP}

Die EFPP wurde 1991 in London gegründet und umfasst heute nationale Gesellschaften in praktisch allen westeuropäischen Ländern mit insgesamt rund 14000 Mitgliedern. Ihr Hauptziel ist die therapeutische Umsetzung und die Verankerung des psychoanalytischen Wissens im öffentlichen Gesundheitswesen (public sector): Psychoanalytische Psychotherapien sollen breitesten Bevölkerungsschichten zugänglich sein.

Die EFPP setzt vier Akzente:

1) Förderung der klinischen Praxis und Forschung durch Kongresse, Tagungen, Publikationen und Forschungsprojekte.

2) Unterstützung und Realisation von Ausbildungsprogrammen für Ausbildungskandidatinnen und -kandidaten, von Weiterbildungsprogrammen für praktizierende Therapeutinnen und Therapeuten.

3) Politische Standortbestimmung und Einflussnahme auf Behörden und Versicherungsträger.

4) Entwicklung und Vertretung von Ausbildungsstandards zur Qualitätssicherung der beruflichen Aus- und Weiterbildung.

In der Schweiz gibt es die folgenden Sektionen der EFPP, die eng zusammenarbeiten und ein nationales Netzwerk bilden:

- Sektion «Psychoanalytische Psychotherapie für Erwachsene» Deutsche Schweiz;

- Sektion «Psychoanalytische Psychotherapie für Kinder und Jugendliche»;

- Sektion «Gruppenanalyse» und Association Romande pour la Psychothérapie Psychanalytique de Groupe (ARPAG); 
- Association Romande pour la Psychothérapie Psychanalytique (ARPP);

- Accademia di Psicoterapia Psicoanalitica della Svizzera Italiana (APPsi).

\section{Die EFPP Sektion Erwachsene Deutsche Schweiz}

Diese Vereinigung versteht sich als Forum für Psychotherapeutinnen und -therapeuten, die auf der Grundlage der Psychoanalytischen Psychotherapie arbeiten, und als Vertreterin der Psychoanalytischen Psychotherapie in der Öffentlichkeit.

Im Zentrum ihrer Aktivitäten stehen der fachliche Austausch und die Verbreitung fachspezifischer Informationen aus dem gesamten Gebiet der Psychoanalytischen Psychotherapie Erwachsener. In der Auseinandersetzung über wissenschaftliche und praxisbezogene Fragen, in der persönlichen Begegnung sowie in der Diskussion mit der Öffentlichkeit wollen wir uns neuen klinischen und sozialen Herausforderungen stellen. Wichtige Akzente setzen dabei unsere Initiativen in Arbeitsgruppen betreffend Öffentlichkeitsarbeit, Forschungsprojekte, Ausbildungsangebote usw., Fachtagungen, Forschung sowie Beteiligungen an internationalen Symposien.

Einen weiteren Schwerpunkt bildet die Verankerung der Psychoanalytischen Psychotherapie in Institutionen und im öffentlichen Gesundheitswesen, insbesondere in der durch die Krankenkassen versicherten Versorgung.

Im Sinne der Qualitätssicherung Psychoanalytischer Psychotherapie vertreten wir die Notwendigkeit hoher Ausbildungsstandards und fördern den Aufbau und die Anerkennung spezifischer Aus- und Weiterbildungen. Als Orientierungsrahmen dienen die «Ausbildungsstandards der EFPP».

Bei Erfüllung der europäisch und schweizerisch anerkannten Ausbildungskriterien besteht die Möglichkeit, sich von der Ausbildungskommission der EFPP als Psychoanalytische Psychotherapeutin/ Psychoanalytischer Psychotherapeut EFPP-zertifizieren zu lassen.

Ziel der Zertifizierung sollte es sein, Kolleginnen und Kollegen, die psychoanalytisch arbeiten, die Möglichkeit zu geben, sich mit die- sem Titel eine Anerkennung zu erwerben, die als Leistungsausweis für ihre psychotherapeutischen Fähigkeiten im Bereich psychoanalytischer Psychotherapie gilt.

Für FMH-Titelträger Psychiatrie und Psychotherapie bedeutet dies einen zusätzlichen Aufwand zur Psychotherapie i.e.S. nach neuem Weiterbildungsprogramm. Für Chefärzte ist das Zertifikat eine Hilfe bei der Beurteilung von Supervisoren und Ausbildnern der Assistenzärztinnen und -ärzte.

Auch wenn man gegenüber der allgemeinen Titelproliferation kritisch eingestellt ist, muss man doch sehen, dass die Schaffung eines Titels zu einem gängigen Instrument, ein Gebiet zu fördern, geworden ist. Die bisherige, im Vergleich zu anderen psychotherapeutischen Richtungen grosse Zurückhaltung psychoanalytischer Institutionen gegenüber Ausbildungszertifikaten hat berechtigte Gründe, vernachlässigt aber das Bedürfnis vieler psychoanalytisch tätiger Kolleginnen und Kollegen, die sich hohen Anforderungen stellen und eine fundierte psychoanalytische Ausbildung erwerben, sich darüber ausweisen zu können.

\section{Fazit}

Die Autoren hoffen, mit ihrem Einsatz für die EFPP dem psychoanalytischen Denken in Weiterund Fortbildung von Psychiatern wieder vermehrt Gewicht geben und gleichzeitig für eine hohe Qualität besorgt sein zu können. Die internationale Vernetzung, die Forschungsanstrengungen und die Anwendung im «public health sector» garantieren, dass die Bemühungen der EFPP für die «Psychoanalytische Psychotherapie» nicht im Elfenbeinturm bleiben, sondern in der sozialen Realität unserer Patienten praktiziert, reflektiert und wissenschaftlich untersucht werden.

Über nationale und internationale Tätigkeiten, Tagungen, Texte, Bücher und Diskussionen berichten regelmässig die Website www.efpp.org und eine Informationsbroschüre.

Information und Antragsformulare sind erhältlich bei D. Leser-Junack, Spiegelbergstrasse 5, 4059 Basel, Tel. 06133182 92, Fax 06133380 31, E-Mail: dleser@bigfoot.com. 